\title{
Quantized Agreement under Time-varying Communication Topology
}

\author{
Dimos V. Dimarogonas and Karl H. Johansson
}

\begin{abstract}
Cooperative control under quantized information for multi-agent systems with continuous models of motion is considered. Time-varying communication topology is taken into account and we distinguish between uniform and logarithmic quantization. Convergence guarantees are provided when the graph is a tree sufficiently often for the logarithmic quantizer, using tools from algebraic graph theory and Lyapunov stability. The results are illustrated by computer simulations.
\end{abstract}

\section{INTRODUCTION}

Multi-agent cooperative control is a field that has gained increasing attention recently, due to the numerous applications that arise from the use of multiple robots/vehicles that cooperate to achieve objectives in a distributed manner. Algorithms for state agreement [4],[15],[5], formation control [1],[17] and flocking motion [18],[20] are some of the results that appeared in recent literature.

Despite most of the results examine the communication topology of the underlying network, an important aspect is that of the quality of the data each agent attains with respect to its neighboring agents' states in order to implement its distributed control law. Therefore, the stability of distributed multi-agent networks under quantized communication is an issue that should be investigated both from an analysis as well as a design perspective. Several results appeared recently that tackle this issue in a distributed manner; these include [10],[6],[3], [12]. A common factor in the aforementioned papers is the use of discrete-time models for the agents' motion. In this paper we use a continuous-time model instead. The only information each agent has is a quantized estimate of the relative position of a subset of the rest of the agents at each time instant. Thus, the need of global coordinates' knowledge is avoided, a requirement imposed by omnidirectional camera sensors that are useful in distributed multi-robot systems [11].

We first treat the static communication topology case with uniform and logarithmic quantizers and show that convergence is achieved in the case of a tree topology. The results are then extended to switching topologies. The stability analysis is held using a Lyapunov approach [14],[2] and the results are supported through computer simulations.

The rest of the paper is organized as follows: Section II presents the problem treated in this paper and provides the background on cooperative control problems with perfect

The authors are with the ACCESS Linnaeus Center, School of Electrical Engineering, Royal Institute of Technology, SE-100 44,Stockholm, Sweden dimos, kallejeee.kth.se. This work was done within the TAISAURES project which is supported by the Swedish Governmental Agency for Innovation Systems (VINNOVA) and Swedish Defence Materiel Administration (FMV). It was also supported by the Swedish Research Council, the Swedish Foundation for Strategic Research, and the EU NoE HYCON. information. In Section III, we treat the case of static communication topology and then tackle the time-varying topology case. The paper concludes with computer simulations in Section IV and a summary of the results in Section V.

\section{System MOdel AND BACKGROUND}

We consider $N$ single integrator agents,

$$
\dot{z}_{i}=u_{i}, i \in\{1, \ldots, N\}
$$

where $z_{i}=\left[x_{i}, y_{i}\right]^{T} \in \mathbb{R}^{2}$ is the position and $u_{i} \in \mathbb{R}^{2}$ the control input of agent $i$.

The design objective is to construct feedback controllers that lead the multi-agent system to agreement, i.e., all agents converge to a common point in $\mathbb{R}^{2}$. Each agent is assigned a subset $\mathcal{N}_{i} \subset\{1, \ldots, N\}$ of the rest of the team, called agent $i$ 's communication set, that includes the agents with which it can communicate. Inter-agent communication can be encoded in terms of an undirected communication graph $G=$ $\{V, E\}$, which consists of a set of vertices $V=\{1, \ldots, N\}$ indexed by the team members, and a set of edges, $E=$ $\left\{(i, j) \in V \times V \mid i \in \mathcal{N}_{j}\right\}$ containing pairs of vertices that represent inter-agent communication specifications.

Each agent only knows the state of agents that belong to its communication set at each time instant. The communication graph is assumed undirected, $i \in \mathcal{N}_{j} \Leftrightarrow j \in \mathcal{N}_{i}, \forall i, j \in$ $\{i, \ldots, N\}, i \neq j$. When the communication topology is static, the sets $\mathcal{N}_{i}$ are static and $G$ is time-invariant. When the communication topology is time-varying, the sets $\mathcal{N}_{i}$ change over time and $G$ is time-varying, i.e., $G=G(t)$.

We will use terminology from algebraic graph theory [8]. For $G=\{V, E\}$, the $N \times N$ adjacency matrix $A=A(G)=$ $\left(a_{i j}\right)$ is given by $a_{i j}=1$, if $(i, j) \in E$ and $a_{i j}=0$, otherwise. If $(i, j) \in E$, then $i, j$ are called adjacent. A path of length $r$ from $i$ to $j$ is a sequence of $r+1$ distinct vertices starting with $i$ and ending with $j$ such that consecutive vertices are adjacent. If there is a path between any two vertices, then $G$ is called connected. A connected graph is called a tree if it contains no cycles. The degree $d_{i}$ of vertex $i$ is $d_{i}=\{\# j:(i, j) \in E\}$. Let $\Delta$ be the $N \times N$ diagonal matrix of $d_{i}$ 's. The Laplacian of $G$ is the symmetric positive semidefinite matrix $L=\Delta-A$. For a connected graph, $L$ has a simple zero eigenvalue and the corresponding eigenvector is the vector of ones. An orientation on $G$ is the assignment of a direction to each edge. The graph $G$ is called oriented if it is equipped with a particular orientation. The incidence matrix $B=B(G)=\left(B_{i j}\right)$ of an oriented graph is the $\{0, \pm 1\}$-matrix with rows and columns indexed by the vertices and edges of $G$, respectively, such that $B_{i j}=1$ if the vertex $i$ is the head of the edge $j, B_{i j}=-1$ if the vertex 
$i$ is the tail of the edge $j$, and 0 otherwise. The Laplacian matrix is also given by $L=B B^{T}=\Delta-A$ [8].

In the sequel, the case of a static communication graph with $m$ edges is treated first and the results are extended to the time-varying case. We denote by $L$ the Laplacian of $G$, by $B$ its incidence matrix corresponding to an arbitrary orientation and by $x=\left[x_{1}, \ldots, x_{N}\right]$ the stack vector for the coordinates of the agents in the $x$-direction. The exact same analysis can be held for the coordinates in the $y$-axis. Moreover, we denote by $\bar{x}$ the $m$-dimensional stack vector of relative differences in the $x$-axis of pairs of agents that form an edge in $G$, where $m$ is the number of edges. The following relations are easily verified: $L x=B \bar{x}, \bar{x}=B^{T} x$. The fact that $\bar{x}=0$ corresponds to agreement is due to that $\bar{x}=0 \Rightarrow B \bar{x}=0 \Rightarrow L x=0$. If $G$ is connected, the last equation guarantees that $x$ has all its elements equal [7],[8].

The agreement control laws in [7], [19] were given by

$$
u_{i}=-\sum_{j \in \mathcal{N}_{i}}\left(x_{i}-x_{j}\right)
$$

and the closed-loop equations of the nominal system (without quantization) were $\dot{x}_{i}=-\sum_{j \in \mathcal{N}_{i}}\left(x_{i}-x_{j},\right), i \in\{1, \ldots, N\}$, so that $\dot{x}=-L x$. Then, $\dot{\bar{x}}=B^{T} \dot{x}=-B^{T} L x=-B^{T} B \bar{x}$. Hence the nominal system is also given by

$$
\dot{\bar{x}}=-B^{T} B \bar{x}
$$

In this paper we impose the additional constraint of quantized relative measurements. Almost all on-board sensors in robotic systems have no access to global coordinates but only measurements of relative states of nearby agents. Moreover, the actuators of each robot can in practice only implement and/or perceive a quantized value of these relative measurements. For these reasons, this paper studies a simplified model of quantized information exchange. In particular, each agent $i$ is assumed to have quantized measurements $q\left(x_{i}-x_{j}\right), q\left(y_{i}-y_{j}\right)$ of the relative position of all of its neighbors $j \in \mathcal{N}_{i}$ where $q():. \mathbb{R} \rightarrow \mathbb{R}$ is the quantization function. The situation is depicted in Figure 1. Each agent $i$

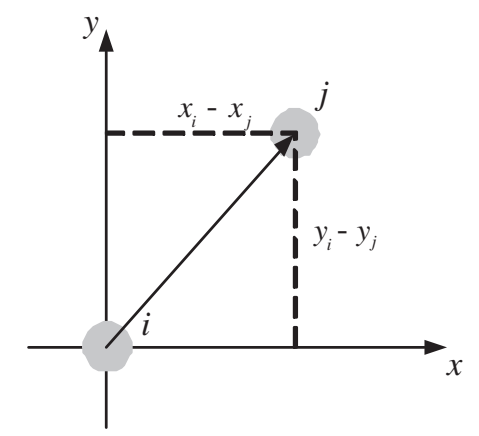

Fig. 1. Each agent $i$ has quantized sensing measurements $x_{i}-x_{j}, y_{i}-y_{j}$ of its relative displacement in the $x$ and $y$ coordinates from all agents $j$ that belong to its communication set $\mathcal{N}_{i}$. Agent $i$ is only aware of a quantized measurement $q\left(x_{i}-x_{j}\right), q\left(y_{i}-y_{j}\right)$ of each of these measurements.

can get estimates of the relative position coordinates $x_{i}-x_{j}$, $y_{i}-y_{j}$ from each of its neighbors $j \in \mathcal{N}_{i}$ using a sensor that can only provide measurements in a quantized way. Since the values of the quantizer are decomposed into the relative measurements $q\left(x_{i}-x_{j}\right), q\left(y_{i}-y_{j}\right)$ in the $x$ and $y$ coordinates respectively, we can treat only the behavior of the system in the $x$ coordinates. The analysis that follows holds mutatis mutandis in the $y$ coordinates, and also in the rest of the coordinates when the agent model is three-dimensional or higher. We hence examine the stability properties of the closed-loop system in the $x$-coordinates under quantization, namely of the system $\dot{x}_{i}=-\sum_{j \in \mathcal{N}_{i}} q\left(x_{i}-x_{j}\right)$, with $i \in$ $\{1, \ldots, N\}$

In this paper, we consider two types of quantized sensors: uniform and logarithmic quantizer. They are given as:

- The uniform quantizer, $q_{u}: \mathbb{R} \rightarrow \mathbb{R}$,

$$
\left|q_{u}(a)-a\right| \leq \delta_{u}, \forall a \in \mathbb{R}
$$

- The logarithmic quantizer $q_{l}: \mathbb{R} \rightarrow \mathbb{R}$,

$$
\left|q_{l}(a)-a\right| \leq \delta_{l}|a|, \forall a \in \mathbb{R}
$$

In the previous equations, $\delta_{u}, \delta_{l}$ are positive scalar gains. We shall use the notation $q($.$) for the quantizer when it is not$ specified if it is a uniform or a logarithmic quantizer.

For a vector $v=\left[v_{1}, \ldots, v_{d}\right] \subset \mathbb{R}^{d}$ of size $d$, the following bounds are easily shown to hold:

- In the uniform quantizer case,

$$
\left|q_{u}(v)-v\right| \leq \delta_{u} \sqrt{d}
$$

- In the logarithmic quantizer case,

$$
\left|q_{l}(v)-v\right| \leq \delta_{l}|v|
$$

\section{QUANTIZED AGREEMENT UNDER TIME-VARYING TOPOLOGY}

In this section, we provide the main results of the paper. We first assume that the communication topology is static, i.e. that the communication sets $\mathcal{N}_{i}$ do not vary over time. A sufficient condition for agreement under quantized information is provided. This simple result is then used to treat the more general case of time-varying communication topology, which is the main contribution of this paper.

\section{A. Static Communication Topology}

In the case of quantized information we have

$$
\dot{x}_{i}=-\sum_{j \in \mathcal{N}_{i}} q\left(x_{i}-x_{j}\right)
$$

where $q():. \mathbb{R} \rightarrow \mathbb{R}$ is the quantizing function. If this function satisfies $q(-a)=-q(a)$ for all $a \in W \subset \mathbb{R}$, which is the case for both types of quantizers used in this paper, then it is easily shown that

$$
\dot{\bar{x}}=-B^{T} B q(\bar{x})
$$

where $q(\bar{x})$ is the stack vector of all pairs $q\left(x_{i}-x_{j}\right)$ with $(i, j) \in E$. While $L$ is always positive semidefinite, the matrix $B^{T} B$ can be either positive semidefinite or positive definite. The next Lemma states that in the case of a tree graph, the matrix $B^{T} B$ is always positive definite: 
Lemma 1: Assume that $G$ is a tree. Then the corresponding matrix $B^{T} B$ is positive definite.

Proof: For arbitrary $y \in \mathbb{R}^{m}$ we have $y^{T} B^{T} B y=|B y|^{2}$ and hence $y^{T} B^{T} B y>0$ if and only if $B y \neq 0$, i.e., $B$ has empty null space. For a connected graph, the cycle space of the graph coincides with the null space of $B$ (Lemma 3.2 in [9]). This corresponds to the fact that for $G$, which has no cycles, zero is not an eigenvalue of $B$. This implies that $\lambda_{\min }\left(B^{T} B\right)>0$, i.e., that $B^{T} B$ is positive definite. $\diamond$

In essence, when the communication graph is a tree, we have $\lambda_{\min }\left(B^{T} B\right)>0$. We use the quadratic edge function

$$
V=\frac{1}{2} \bar{x}^{T} \bar{x}
$$

as a candidate Lyapunov function. Assuming that the communication graph is a tree, the derivative of $V=\frac{1}{2} \bar{x}^{T} \bar{x}$ along the trajectories of the closed loop system (7) is given by

$$
\dot{V}=-\bar{x}^{T} B^{T} B q(\bar{x})=-\bar{x}^{T} B^{T} B \bar{x}-\bar{x}^{T} B^{T} B(q(\bar{x})-\bar{x})
$$

so that

$$
\dot{V} \leq-\lambda_{\min }\left(B^{T} B\right)|\bar{x}|^{2}-\bar{x}^{T} B^{T} B(q(\bar{x})-\bar{x})
$$

In the case of a uniform quantizer we have $q=q_{u}$ and $\left|q_{u}(\bar{x})-\bar{x}\right| \leq \delta_{u} \sqrt{m}$, where $m$ is the number of edges in the communication graph. Then (9) yields

$$
\begin{aligned}
& \dot{V} \leq-\lambda_{\min }\left(B^{T} B\right)|\bar{x}|^{2}+|\bar{x}|\left\|B^{T} B\right\| \delta_{u} \sqrt{m} \\
& \leq-\lambda_{\min }\left(B^{T} B\right)|\bar{x}|\left(|\bar{x}|-\frac{\left\|B^{T} B\right\| \delta_{u} \sqrt{m}}{\lambda_{\min }\left(B^{T} B\right)}\right)
\end{aligned}
$$

Thus, all solutions of the closed-loop system enter the ball

$$
\left\{x:|\bar{x}| \leq \frac{\left\|B^{T} B\right\| \delta_{u} \sqrt{m}}{\lambda_{\min }\left(B^{T} B\right)}\right\}
$$

centered at $\bar{x}=0$ of radius $\frac{\left\|B^{T} B\right\| \delta_{u} \sqrt{m}}{\lambda_{\min }\left(B^{T} B\right)}$ in finite time.

In the case of a logarithmic quantizer we have $q=q_{l}$ and $\left|q_{l}(\bar{x})-\bar{x}\right| \leq \delta_{l}|\bar{x}|$ and (9) yields

$$
\dot{V} \leq-\lambda_{\min }\left(B^{T} B\right)|\bar{x}|^{2}+\left\|B^{T} B\right\| \delta_{l}|\bar{x}|^{2},
$$

so that

$$
\dot{V} \leq-|\bar{x}|^{2}\left(\lambda_{\min }\left(B^{T} B\right)-\left\|B^{T} B\right\| \delta_{l}\right)
$$

Convergence to an agreement point $\bar{x}=0$ is guaranteed for

$$
\delta_{l}<\frac{\lambda_{\min }\left(B^{T} B\right)}{\left\|B^{T} B\right\|}
$$

i.e. the gain $\delta_{l}$ of the logarithmic quantizer must be sufficiently small. The fact that $\bar{x}=0$ guarantees that the vector $x$ has all its elements equal, in the case of a connected graph.

By applying the Comparison Lemma [13] in equation (10) we get the following estimates of the convergence rate for the case of a logarithmic quantizer and a tree structure:

$$
V(\bar{x}(t)) \leq e^{-2\left(\lambda_{\min }\left(B^{T} B\right)-\left\|B^{T} B\right\| \delta_{l}\right) t} V(\bar{x}(0))
$$

so that

$$
\bar{x}(t) \leq e^{-\left(\lambda_{\min }\left(B^{T} B\right)-\left\|B^{T} B\right\| \delta_{l}\right) t} \bar{x}(0)
$$

for all times $t \geq 0$.

The previous derivations yield the following Theorem for the time invariant communication topology case :

Theorem 2: Assume that the time-invariant communication graph $G$ is a tree. Then the closed loop system (7) has the following convergence properties:

- In the case of a uniform quantizer, the system converges to a ball of radius $\frac{\left\|B^{T} B\right\| \delta_{u} \sqrt{m}}{\lambda_{\min }\left(B^{T} B\right)}$ which is centered in the desired equilibrium point $\bar{x}=0$ in finite time.

- In the case of a logarithmic quantizer, the system is exponentially stabilized to an agreement point $\bar{x}=0$, provided that the gain of the quantizer $\delta_{l}$ satisfies (11).

Using now (10) we get the following useful relations for the trajectories of the closed loop system in the general case when the communication graph is not necessarily a tree:

$$
V(\bar{x}(t)) \leq e^{2\left\|B^{T} B\right\| \delta_{l} t} V(\bar{x}(0))
$$

so that

$$
\bar{x}(t) \leq e^{\left\|B^{T} B\right\| \delta_{l} t} \bar{x}(0)
$$

The previous equations will be used in the time-varying communication topology network analyzed in the sequel.

\section{B. Main Result: Time-varying Communication Topology}

In this section we treat the case when the communication topology is time-varying, allowing each agent to lose/create new communication links with other agents as the closedloop system evolves. The problem in this case is that it's not possible to use $V=\frac{1}{2} \bar{x}^{T} \bar{x}$ as a common Lyapunov function for the switched system, since the vector $\bar{x}$ changes discontinuously whenever edges are added or deleted when the communication topology changes. A different energy function is used and in particular, the function

$$
W=\max \left\{x_{1}, \ldots, x_{N}\right\}-\min \left\{x_{1}, \ldots, x_{N}\right\}
$$

which can act as a common Lyapunov function for the switched system.

Let $x_{\max } \triangleq \max \left\{x_{1}, \ldots, x_{N}\right\}, x_{\min } \triangleq$ $\min \left\{x_{1}, \ldots, x_{N}\right\}$ denote the maximum and minimum element of $x$, respectively. In the degenerate case that more than one elements is equal to the maximum element or minimum element, we define $x_{\max } \triangleq x_{m_{1}}$ and $x_{\min } \triangleq x_{m_{2}}$ where $m_{1} \triangleq \max _{i}\left\{i: x_{i}=\max \left\{x_{1}, \ldots, x_{N}\right\}\right\}$ and $m_{2} \triangleq \min _{i}\left\{i: x_{i}=\min \left\{x_{1}, \ldots, x_{N}\right\}\right\}$.

The notation $\mathcal{T}=\left\{t_{1}, \ldots, t_{j}, \ldots\right\}$ is used for the set of switching instants, i.e., times when a new communication link is created or an existing one is lost, or the maximum or minimum element change, i.e., a new agent attains the maximum or minimum value, $x_{\max }$ or $x_{\min }$, respectively. We will use the extension of LaSalle's Invariance Principle for hybrid systems [16] to check the stability of the overall system. The main result is stated as follows:

Theorem 3: Assume that the time-varying communication graph $G=G(t)$ remains a tree for all continuous evolution intervals $\left[t_{i}, t_{i+1}\right]$ and the quantizer is logarithmic. Then the 
system converges to an agreement point, provided that the gain of the logarithmic quantizer $\delta_{l}$ satisfies

$$
\delta_{l}<\min _{B \in T(B)} \frac{\lambda_{\min }\left(B^{T} B\right)}{\left\|B^{T} B\right\|}
$$

where the minimization is held over all possible incidence matrices that belong to the set $T(B)$ of incidence matrices corresponding to all possible trees with $N$ vertices.

Proof: We have to show that $W$ is strictly decreasing in between arbitrary switching instances. For the logarithmic quantizer we have $\operatorname{sign}\left(q_{l}(x)\right)=\operatorname{sign}(x)$. Since $x_{\max } \geq x_{i}$ and $x_{\min } \leq x_{i}$ for all $i \in[1, \ldots, N]$, the following equations hold for all $t \in\left[t_{i}, t_{i+1}\right]$, for a time interval $\left[t_{i}, t_{i+1}\right]$, where $t_{i}, t_{i+1} \in \mathcal{T}: \dot{x}_{\max }=-\sum_{j \in \mathcal{N}_{\max }} q_{l}\left(x_{\max }-x_{j}\right) \leq 0$, and $\dot{x}_{\text {min }}=-\sum_{j \in \mathcal{N}_{\max }} q_{l}\left(x_{\min }-x_{j}\right) \geq 0$.

The previous calculations prove that $W$ is non-increasing throughout the closed loop system evolution. We now show that $W$ is strictly decreasing within each subinterval $[\tau, \tau+$ $\Delta \tau]$ of $\left[t_{i}, t_{i+1}\right]$ with non-zero measure as long as the communication graph is a tree and the system has not reached an agreement point $\bar{x}=0$. This is proved by contradiction. Assume first that $x_{\max }$ is constant at each time instant the time interval in consideration, i.e. $\dot{x}_{\max }=0$, for all $t \in$ $[\tau, \tau+\Delta \tau]$. This is equivalent to $\sum_{j \in \mathcal{N}_{\max }} q_{l}\left(x_{\max }-x_{j}\right)=0$, and since $x_{\max } \geq x_{i}$ for all $i \in\{1, \ldots, N\}$ the latter implies that $x_{j}=x_{\max }$ for all $j \in \mathcal{N}_{\max }$.

Pick a random $k \in \mathcal{N}_{\max }$, where $k$ does not coincide with the maximum vertex. Then $x_{k} \geq x_{j}$, for all $j \in \mathcal{N}_{k}$ and hence $\dot{x}_{k}=-\sum_{j \in \mathcal{N}_{k}} q_{l}\left(x_{k}-x_{j}\right) \leq 0$. If $\dot{x}_{k}<0$, then necessarily $\dot{x}_{\max }<0$ since $x_{k}=x_{\max }$ for all $t \in[\tau, \tau+\Delta \tau]$. Hence we also have $\dot{x}_{k}=0$ and hence $x_{j}=x_{k}=x_{\max }$ for all $j \in \mathcal{N}_{k}$. We can now repeat the same procedure for a random $l \in \mathcal{N}_{k}$. Since the graph is a tree and has finite vertices, we conclude that there exists a finite number of iterations of the above procedure that propagates to every vertex in the graph. We hence conclude that all vertices in the graph should have a zero time derivative. By virtue of the above procedure all vertices then will have a common value equal to the constant maximum value of $x_{\max }$. This is of course a contradiction to the fact that function $V$ is strictly decreasing, by virtue of (10),(11),(17), as long as the system has not reached an agreement point. We therefore conclude that using the above procedure, there should be at least one vertex $p$ chosen in the above iterative procedure which necessarily has a strictly negative time derivative at some $t \in[\tau, \tau+\Delta \tau]$. Since the above procedure suggests that $x_{p}=x_{\max }$, and therefore $\dot{x}_{p}=\dot{x}_{\max }$, for all $t \in[\tau, \tau+\Delta \tau]$, we conclude that $x_{\max }$ is strictly decreasing in $[\tau, \tau+\Delta \tau]$.

The above analysis can be used to show -albeit not necessary for our proof- that $x_{\min }$ is strictly increasing in $[\tau, \tau+\Delta \tau]$. We conclude that $W$ strictly decreases within each time interval $\left[t_{i}, t_{i+1}\right]$, i.e. $W\left(t_{i}\right)<W\left(t_{i+1}\right)$. We conclude that $W$ converges to its minimum value of zero as $t \rightarrow \infty$. The latter of course corresponds to a desired agreement point by definition. This completes the proof. $\diamond$

\section{The Case when Connectedness is lost in some Intervals}

The above result is useful whenever the communication topology retains the tree structure at all switching instances. A more practical situation however occurs if we allow for the tree assumption to be lost for some times. In particular, we assume that in between moments where the team switches to a different tree structures, there are time intervals where the connected tree assumption is not guaranteed to hold. Hence we consider a switching sequence of the form $\mathcal{T}=\{0=$ $\left.t_{01}, t_{1}, t_{12}, t_{2}, t_{23}, t_{3}, \ldots\right\}$, where intervals of the form $\Delta t_{i}=$ $t_{i}-t_{i-1, i}$ correspond to a tree communication graph while the reset intervals $\Delta t_{i, i+1}=t_{i, i+1}-t_{i}$ correspond to the a switch between two trees. The connectivity assumption is not guaranteed to hold in the reset intervals $\Delta t_{i, i+1}$. Figure 2 shows a possible evolution of the communication topology.

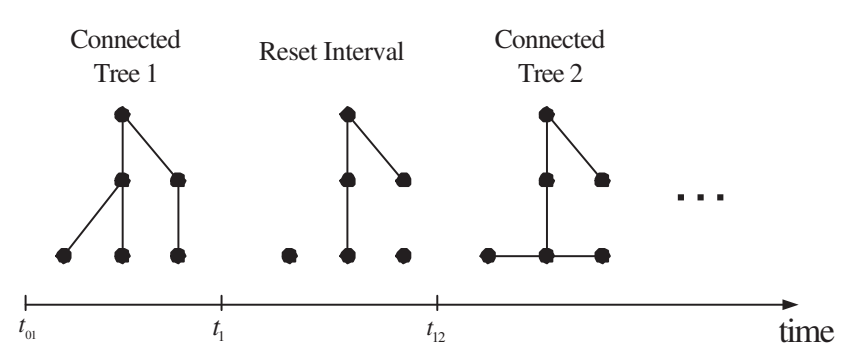

Fig. 2. A switching scenario. Between the two tree structures there is a reset interval $\Delta t_{12}=t_{12}-t_{1}$ where connectivity is lost.

We assume that each time interval $\Delta t_{i}$ where the communication topology is a tree has a minimum dwell time $\Delta t_{\text {min }}$, i.e. $\Delta t_{i}>\Delta t_{\text {min }}$. The following result states that convergence to a rendezvous point can still be achieved provided that the reset intervals are chosen small enough:

Theorem 4: Assume that the time-varying communication graph $G=G(t)$ is a tree for all time intervals $\Delta t_{i}=t_{i}-$ $t_{i-1, i}$ and the quantizer is logarithmic. Further assume that there is a path connecting the maximum and the minimum vertex, for all reset time intervals of the form $\Delta t_{i, i+1}=$ $t_{i, i+1}-t_{i}$. Assume that there exists an $\varepsilon$, where $0<\varepsilon<$ $\min _{B \in T(B)} \frac{\lambda_{\min }\left(B^{T} B\right)}{\left\|B^{T} B\right\|}$, such that the quantizer gain satisfies

$$
\delta_{l}<\min _{B \in T(B)} \frac{\lambda_{\min }\left(B^{T} B\right)}{\left\|B^{T} B\right\|}-\varepsilon
$$

Furthermore, assume that the tree time intervals $\Delta t_{i}$ satisfy

$$
\Delta t_{\min }>\frac{2 \ln (N(N-1) / 2)}{\varepsilon \cdot \max _{B \in T(B)}\left\|B^{T} B\right\|}
$$

Then the closed-loop system converges to agreement, provided that the reset time intervals $\Delta t_{i, i+1}$ are sufficiently smaller than an upper bound which is provided in the proof. Proof: We consider $W_{c}=\frac{W}{\sqrt{N(N-1)}}$ as a common Lyapunov function for the overall switched system. Since for all intervals there is a path $\max , p_{1}, p_{2}, \ldots, p_{f}$, min connecting the maximum and minimum vertices, we have $W=x_{\max }-$ 
$x_{\min }=x_{\max }-x_{p_{1}}+x_{p_{1}}-x_{p_{2}}+\ldots+x_{p_{f}}-x_{\min }$, and using the inequality $n \sum_{i=1}^{n} r_{i}^{2} \geq\left(\sum_{i=1}^{n} r_{i}\right)^{2}, \forall r_{i} \in \mathbb{R}$, we have

$$
\begin{aligned}
& W^{2} \leq \frac{N(N-1)}{2} \\
& \cdot\left[\left(x_{\max }-x_{p_{1}}\right)^{2}+\left(x_{p_{1}}-x_{p_{2}}\right)^{2} \ldots\left(x_{p_{f}}-x_{\min }\right)^{2}\right] \\
& \leq \frac{N(N-1)}{2} 2 V
\end{aligned}
$$

and hence $W_{c} \leq \sqrt{V}$ where $V$ is the quadratic function (8) corresponding to the edges $G(t)$ at each time instant and $N(N-1) / 2$ is the maximum number of edges at each time instant. Hence the candidate common Lyapunov function is bounded from above by $V$ at each time instant, where $V$ corresponds to the vector $\bar{x}$ of edges at the same time instant.

All pairs $i, j \in\{1, \ldots, N\}$ satisfy $\left|x_{\max }-x_{\min }\right| \geq$ $\left|x_{i}-x_{j}\right| \quad$ and thus, $\frac{m}{2}\left(x_{\max }-x_{\min }\right)^{2} \geq$ $\frac{1}{2} \sum_{(i, j) \in E}\left(x_{i}-x_{j}\right)^{2}=V$. Since the maximum number of edges $m$ is $N(N-1) / 2$ the last equation implies $W \geq \frac{2}{\sqrt{N(N-1)}} \sqrt{V} \Rightarrow W_{c} \geq \frac{2}{N(N-1)} \sqrt{V}$. We hence have

$$
\frac{2}{N(N-1)} \sqrt{V} \leq W_{c} \leq \sqrt{V}
$$

for all possible quadratic edge function $V$ corresponding either to a connected tree interval or a reset interval.

With a slight abuse of notation, we denote by $V_{i}$ the quadratic edge function $V$ corresponding to a random tree that represents the communication topology in the time interval $\Delta t_{i}$ and by $V_{i, i+1}$ the quadratic edge function $V$ corresponding to the reset time interval $\Delta t_{i, i+1}$. We now use the bounds derived in (12),(13), (14),(15) to show that for sufficiently small reset time intervals the result still holds.

For two consecutive random intervals $\left[t_{i}, t_{i, i+1}\right],\left[t_{i, i+1}, t_{i+1}\right]$, using equations (12),(14) and the bounds (20) we have

$$
\begin{aligned}
& W_{c}\left(t_{i+1}\right) \leq \sqrt{V_{i+1}\left(t_{i+1}\right)} \\
& \leq e^{-\left(\lambda_{\min }\left(B_{i+1}^{T} B_{i+1}\right)-\left\|B_{i+1}^{T} B_{i+1}\right\| \delta_{l}\right) \Delta_{t_{i+1}}} \sqrt{V_{i+1}\left(t_{i, i+1}\right)} \\
& \leq e^{-\left(\lambda_{\min }\left(B_{i+1}^{T} B_{i+1}\right)-\left\|B_{i+1}^{T} B_{i+1}\right\| \delta_{l}\right) \Delta_{t_{i+1}} \frac{N(N-1)}{2}} \\
& \cdot W_{c}\left(t_{i, i+1}\right) \\
& \leq e^{-\left(\lambda_{\min }\left(B_{i+1}^{T} B_{i+1}\right)-\left\|B_{i+1}^{T} B_{i+1}\right\| \delta_{l}\right) \Delta_{t_{i+1}}} \\
& \cdot \frac{N(N-1)}{2} \sqrt{V_{i, i+1}\left(t_{i, i+1}\right)} \\
& \leq e^{-\left(\lambda_{\min }\left(B_{i+1}^{T} B_{i+1}\right)-\left\|B_{i+1}^{T} B_{i+1}\right\| \delta_{l}\right) \Delta_{t_{i+1}} \frac{N(N-1)}{2}} \\
& \cdot e^{\left\|B_{i, i+1}^{T} B_{i, i+1}\right\| \delta_{l} \Delta_{t_{i, i+1}}} \sqrt{V_{i, i+1}\left(t_{i}\right)} \\
& \leq\left(\frac{N(N-1)}{2}\right)^{2} e^{-\left(\lambda_{\min }\left(B_{i+1}^{T} B_{i+1}\right)-\left\|B_{i+1}^{T} B_{i+1}\right\| \delta_{l}\right) \Delta_{t_{i+1}}} \\
& \cdot e^{\left\|B_{i, i+1}^{T} B_{i, i+1}\right\| \delta_{l} \Delta_{t_{i, i+1}} W_{c}\left(t_{i}\right)}
\end{aligned}
$$

where, in accordance with the defined notation, $B_{i+1} \in$ $T(B)$ is an incidence matrix belonging to the set $T(B)$ of incident matrices corresponding to trees with $N$ vertices, while $B_{i, i+1}$ is an arbitrary incidence matrix corresponding to a graph with $N$ vertices. It suffices to show that $W_{c}$ strictly decreases in the time interval $t_{i}, t_{i+1}$.
This is equivalent to

$$
\begin{aligned}
& \left\{\begin{array}{l}
-\left(\lambda_{\min }\left(B_{i+1}^{T} B_{i+1}\right)-\left\|B_{i+1}^{T} B_{i+1}\right\| \delta_{l}\right) \Delta_{t_{i+1}} \\
+\left\|B_{i, i+1}^{T} B_{i, i+1}\right\| \delta_{l} \Delta_{t_{i, i+1}}
\end{array}\right\} \\
& <\left(\frac{N(N-1)}{2}\right)^{-2} \Leftrightarrow \\
& \Leftrightarrow-\left(\lambda_{\min }\left(B_{i+1}^{T} B_{i+1}\right)-\left\|B_{i+1}^{T} B_{i+1}\right\| \delta_{l}\right) \Delta_{t_{i+1}} \\
& +\left\|B_{i, i+1}^{T} B_{i, i+1}\right\| \delta_{l} \Delta_{t_{i, i+1}}<-2 \ln \left(\frac{N(N-1)}{2}\right)
\end{aligned}
$$

Using $\Delta t_{i+1}>\Delta t_{\min }$, an upper bound on the reset interval time for which the above inequality holds is given by

$$
\Delta_{t_{i, i+1}}<\frac{v_{i+1} \Delta t_{\min }-2 \ln (N(N-1) / 2)}{\left\|B_{i, i+1}^{T} B_{i, i+1}\right\| \delta_{l}}
$$

where the parameter $v_{i+1}=\lambda_{\min }\left(B_{i+1}^{T} B_{i+1}\right)-$ $\left\|B_{i+1}^{T} B_{i+1}\right\| \delta_{l}$ is always positive, due to $\delta_{l}$ satisfying (18). Due to the fact that $\Delta t_{\min }$ satisfies (19), there is a strictly positive upper bound on the reset intervals $\Delta t_{\max }^{r}$ for which (21) holds, i.e. we have $\Delta t_{i, i+1}<\Delta t_{\max }^{r}$ for all $i$, and

$$
\Delta t_{\max }^{r}<\min _{i} \frac{v_{i+1} \Delta t_{\min }-2 \ln (N(N-1) / 2)}{\left\|B_{i, i+1}^{T} B_{i, i+1}\right\| \delta_{l}}
$$

Hence for sufficiently small reset intervals, $W_{c}$ is strictly decreasing, i.e., $W_{c}\left(t_{i+1}\right)<W_{c}\left(t_{i}\right)$ for all $i$. The result follows by allowing $i$ tend to infinity. $\diamond$

The above result shows that convergence can be achieved in the presence of the reset intervals, provided that the tree intervals, and the quantizer gain are appropriately tuned. If the latter is sufficiently small, the reset intervals are allowed to be large enough provided that the tree intervals have a sufficiently large lower bound $\Delta t_{\text {min }}$. We should also point out here that the requirement that there is a path connecting the maximum and minimum vertex is rather conservative. The means to relax this condition are under investigation. Simulation results verify the fact that this condition is far from necessary. On the other hand, Theorem 4 shows that the closed loop system is robust in terms of temporary lack of connectivity and quantization effects.

\section{Simulations}

We provide simulations to support the presented theory.

The first simulation involves four agents navigating under quantized communication and under a static tree structure. In fact, the communication sets of the four agents are chosen as $\mathcal{N}_{1}=\{2\}, \mathcal{N}_{2}=\{1,3\}, \mathcal{N}_{3}=\{2,4\}, \mathcal{N}_{4}=\{3\}$, so that the corresponding graph is a line graph. We can compute $\frac{\lambda_{\min }\left(B^{T} B\right)}{\left\|B^{T} B\right\|}=0.1716$ in this case. We choose $\delta_{l}=0.15$ and $\delta_{s}=0.1$ in the simulation of Figure 3. The trajectories corresponding to the uniform quantizer control law are depicted by the grey lines and shown on the left screenshot, while the corresponding ones of the logarithmic quantizer by the black lines and shown on the right screenshot. The initial conditions of the four agents are denoted by a cross. As expected, the uniform quantizer only achieves set convergence while the logarithmic one drives the agents to agreement, since condition (11) is fulfilled.

The second simulation involves a switching topology case for the logarithmic quantizer. We allowed the tree structure 

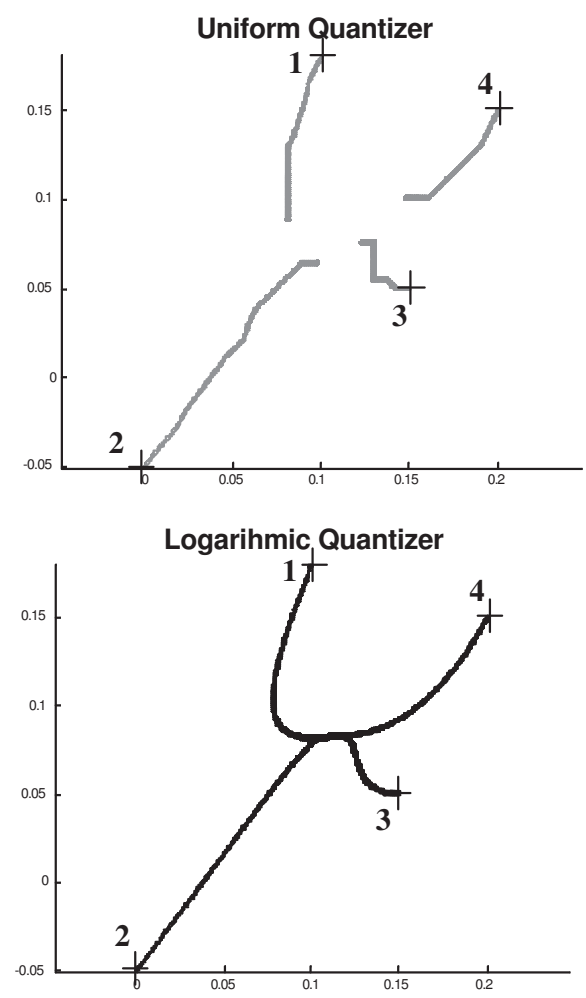

Fig. 3. Uniform vs. Logarithmic quantizer. In the first case only set convergence is guaranteed while in the second case agreement is achieved, since $\delta_{l}$ satisfies (11).

to be lost during some time intervals, and thus the simulation involves the development where connectedness is lost during some time intervals in Section III-C. The agents start from the same initial conditions as in the previous simulations. The reset time intervals are sufficiently small and the logarithmic quantizer gain satisfies (18). Agreement is eventually achieved in Figure 4 since Theorem 4 holds. The nonsmoothness of the trajectories with respect to the previous simulation is due to the topology change.

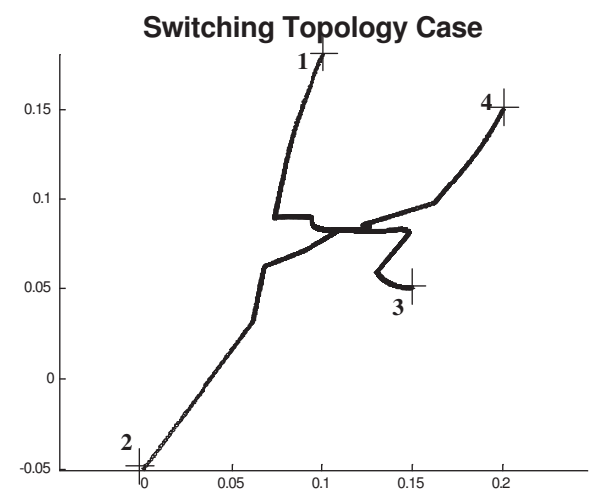

Fig. 4. Agreement with logarithmic quantizer and switching communication topology. Connectedness is lost during the reset intervals. Agreement is reached by virtue of Theorem 4 .

\section{CONCLUSIONS}

Distributed cooperative control laws for multi-agent systems under imperfect, quantized, relative information between neighboring agents were considered. We distinguished between uniform and logarithmic quantizers as well as between static and time-varying communication topologies and showed that a tree structure provides convergence guarantees in both cases. The results were also shown to hold in the case where connectedness is lost during bounded time intervals. Computer simulations supported the derived theory.

\section{REFERENCES}

[1] M. Arcak. Passivity as a design tool for group coordination. IEEE Transactions on Automatic Control, 52(8):1380-1390, 2007.

[2] F. Bullo and D. Liberzon. Quantized control via locational optimization. IEEE Transactions on Automatic Control, 51(1):2-13, 2006.

[3] R. Carli, F. Fagnani, and S. Zampieri. On the state agreement with quantized information. 17th Intern. Symp. Networks and Systems, pages 1500-1508, 2006.

[4] J. Cortes, S. Martinez, and F. Bullo. Robust rendezvous for mobile autonomous agents via proximity graphs in arbitrary dimensions. IEEE Transactions on Automatic Control, 51(8):1289-1298, 2006.

[5] D.V. Dimarogonas and K.J. Kyriakopoulos. On the rendezvous problem for multiple nonholonomic agents. IEEE Transactions on Automatic Control, 52(5):916-922, 2007.

[6] F. Fagnani, K. H. Johansson, A. Speranzon, and S. Zampieri. On multi-vehicle rendezvous under quantized communication. 16th Intern. Symp. Networks and Systems, 2004.

[7] J.A. Fax and R.M. Murray. Graph Laplacians and stabilization of vehicle formations. 15th IFAC World Congress, 2002.

[8] C. Godsil and G. Royle. Algebraic Graph Theory. Springer Graduate Texts in Mathematics \# 207, 2001.

[9] S. Guattery and G.L. Miller. Graph embeddings and laplacian eigenvalues. SIAM Journ. Matrix Anal. Appl., 21(3):703-723, 2000.

[10] K. H. Johansson, A. Speranzon, and S. Zampieri. On quantization and communication topologies in multi-vehicle rendezvous. 16th IFAC World Congress, 2005. electronic proceedings.

[11] M.Mazo Jr., A.Speranzon, K. H. Johansson, and X.Hu. Multi-robot tracking of a moving object using directional sensors. 2004 IEEE International Conference on Robotics and Automation, 2004.

[12] A. Kashyap, T. Basar, and R. Srikant. Quantized consensus. Automatica, 43(7):1192-1203, 2007.

[13] H. Khalil. Nonlinear Systems. Prentice-Hall, 2002.

[14] D. Libezon. Switching in Systems and Control. Birkhäuser, Boston, 2003.

[15] Z. Lin, B. Francis, and M. Maggiore. State agreement problem for continuous-time coupled nonlinear systems. SIAM Journal on Control and Optimization, 46(1):288-307, 2007.

[16] J. Lygeros, K.H. Johansson, S. Simic, J. Zhang, and S. Sastry. Dynamical properties of hybrid automata. IEEE Transactions on Automatic Control, 48(1):2-17, 2003.

[17] A. Muhammad and M. Egerstedt. Connectivity graphs as models of local interactions. Journal of Applied Mathematics and Computation, 168(1):243-269, 2005.

[18] R. Olfati-Saber. Flocking for multi-agent dynamic systems: Algorithms and theory. IEEE Transactions on Automatic Control, 51(3):401-420, 2006

[19] R. Olfati-Saber and R.M. Murray. Consensus problems in networks of agents with switching topology and time-delays. IEEE Transactions on Automatic Control, 49(9):1520-1533, 2004.

[20] H.G. Tanner, A. Jadbabaie, and G.J. Pappas. Flocking in fixed and switching networks. IEEE Transactions on Automatic Control, 52(5):863-868, 2007 